\title{
Niveles de actividad física y sedentarismo en personal de la salud.
}

\author{
Levels of physical activity and \\ sedentarism in health workers.
}

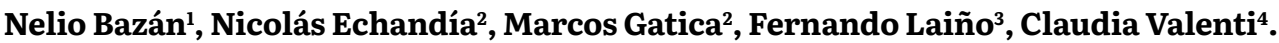 \\ ${ }^{1}$ Universidad Nacional de Rosario. Rosario; Argentina. \\ ${ }^{2}$ Universidad Nacional de Villa Mercedes (UNViMe). Córdoba; Argentina. \\ ${ }^{3}$ Fundación Instituto Superior de Ciencias de la Salud. Buenos Aires; Argentina. \\ ${ }^{4}$ Ministerio de Salud del Gobierno de la Ciudad de Buenos Aires. Buenos Aires; Argentina.
}

Autor por correspondencia: Nelio Bazán — nelio.bazan@gmail.com

Conflicto de intereses: no presenta.

\section{Resumen}

Introducción: la actividad física reduce el riesgo de padecer afecciones cardíacas, diabetes y permite controlar el peso, la salud osteomuscular, aportando beneficios psicológicos. El personal de salud es importante para asesorar sobre actividad física. Resulta interesante conocer si cumplen con las recomendaciones actuales.

Objetivo: estudiar la actividad física, tiempo sedentario, colesterol, glucemia en ayunas y estado nutricional de profesionales de salud.

Métodos: se estudiaron 322 trabajadores de salud de ambos sexos de hospitales de Buenos Aires. Se implementó un cuestionario de actividad física y se estudió la glucemia en ayunas, colesterol, edad, sexo, peso y talla.

Resultados: la edad promedio fue 40.7 años, $40 \%$ no realiza actividad física suficiente. Los activos poseen un mejor estado nutricional $(\mathrm{p}=0.041)$, y alto porcentaje de niveles saludables de colesterol (73.5\%). El tiempo sedentario promedio fue 347.83 minutos, $67 \%$ reporto más de 4 horas.

Discusión: Que el personal de la salud realice actividad física es importante, entre ellos, los médicos deberían formarse sobre el tema durante sus estudios de grado. La promoción de hábitos adecuados de actividad física durante su educación es importante para mejorar el asesoramiento que proporcionaran luego. Se puede ser activo, cumplir con recomendaciones de actividad física, y también ser sedentario, si se queda varias horas sentado. Las pausas activas son útiles para contrarrestar este fenómeno, su implementación debiera ser normativa.

Conclusiones: aproximadamente el 60\% del grupo de profesionales realiza actividad física en niveles recomendados, pero la conducta sedentaria es elevada. Se podrían implementar políticas que contribuyan a disminuir el tiempo sedentario.

Palabras clave: Personal de salud. Tiempo sedentario. Estado nutricional. Colesterol.

\footnotetext{
Abstract

Introduction: physical activity reduces the risk of heart disease, diabetes and allows weight control, musculoskeletal health, providing psychological benefits. Health staff is important to advise on physical activity. It is interesting to know if they comply with the current recommendations.

Objective: to study the physical activity, sedentary time, cholesterol, fasting blood glucose and nutritional status of health professionals.
} 
Methods: 322 health workers of both sexes from hospitals in Buenos Aires were studied. A physical activity questionnaire was implemented, and fasting blood glucose, cholesterol, age, sex, weight and height were studied.

Results: the average age was 40.7 years, $40 \%$ did not perform enough physical activity. The assets have a better nutritional status $(\mathrm{p}=0.041)$, and a high percentage of healthy cholesterol levels $(73.5 \%)$. The average sedentary time was 347.83 minutes, $67 \%$ reported more than 4 hours.

Discussion: That the health professional perform physical activity is important, and should be trained during their undergraduate studies. The promotion of adequate physical activity habits during their education is important to improve the advice they will provide later. You can be active, comply with physical activity recommendations, and also be sedentary, if you have several hours sitting. Active pauses are useful to counteract this phenomenon, its implementation should be normative.

Conclusions: approximately $60 \%$ of the group of professionals performs physical activity at recommended levels, but sedentary behavior is high. Policies could be implemented that help reduce sedentary time.

Keywords: Health workers. Sedentary time. Nutritional status. Cholesterol.

\section{Introducción}

La actividad física (AF) reduce el riesgo de padecer afecciones cardíacas, diabetes y algunos tipos de cáncer, ayuda a controlar el peso, mejora la salud ósea y muscular, y presenta indudables beneficios psicológicos (1). Por ello es conveniente que un estilo de vida saludable incluya actividades físicas, y si estas son diarias, mejor. Los adultos y adultos mayores que mantienen adecuados niveles de AF tienen menor probabilidad de padecer enfermedades crónicas o una muerte prematura (2). Es decir, la AF contribuye a reducir la morbi- mortalidad y a mejorar su calidad de vida por medio de beneficios fisiológicos, psicológicos y sociales (3). Se pueden considerar también los costos económicos de las enfermedades crónicas relacionadas con una insuficiente AF. Se calcula que los costos de salud de las personas activas son un 30 \% inferior a los que ocasionan aquellas inactivas, a lo que se debe agregar el aumento del ausentismo laboral. Se considera que la insuficiente AF es el cuarto factor de riesgo de mortalidad más importante a nivel mundial y provoca el $6 \%$ de todas las muertes. Solo sobrepasada por el $13 \%$ de la hipertensión arterial y el $9 \%$ del consumo de tabaco y conlleva el mismo nivel de riesgo que la hiperglucemia (4). Aproximadamente 3.2 millones de personas mueren cada año por tener un nivel insuficiente de AF, que continúa aumentando en muchos países. Las personas con un nivel insuficiente de AF tienen entre el $20 \%$ y el $30 \%$ más de riesgo de muerte que las personas que realizan al menos 30 minutos de AF moderada la mayoría de días de la semana $(4,5)$. Aunque los 30 minutos de actividad física diaria parecen ser importantes, las evidencias científicas muestran que incluso pueden ser realizados de forma continua o acumulando etapas de 10 o 15 minutos (6).

El descenso de la AF se debe en parte al sedentarismo prevalente en las actividades del tiempo de ocio y en el trabajo. Lo mismo sucede con el transporte. Se viaja sentado en el automotor o en el bus de transporte. Así es que al menos un 60 \% de la población mundial no realiza la AF necesaria para obtener beneficios para la salud (7). El sedentarismo, es decir, el tiempo despierto dedicado a conductas sedentes, es considerado actualmente un factor de elevada morbilidad y mortalidad (8). Los profesionales de la salud promocionan y promueven hábitos saludables para todas las edades. El tiempo que pasa una persona sentada constituye por sí solo un factor de riesgo para la salud, pues puede favorecer enfermedades como la aterosclerosis, la osteoporosis, la hipertensión arterial, la diabetes mellitus y la obesidad, además de los procesos degenerativos propios del envejecimiento (7).

La AF es importante para preservar la salud, y debe ser realizada de forma adecuada, debiendo tenerse en cuenta que los ejercicios deben ser orientados y dosificados adecuadamente. Si bien es cierto que la insuficiente AF es perjudicial, también puede serlo su práctica incorrecta (9). Los ámbitos en los que se puede desarrollar la AF podrían ser el escolar, el transporte o el de la recreación. Lo importante es su papel al evitar la aparición de enfermedades hipocinéticas o bien, participar en su tratamiento. La AF puede provocar adaptaciones orgánicas consideradas benéficas frente a estas enfermedades. La AF se valora en la medida en que provoca dichas adaptaciones, es decir, en la medida en que mejora o mantiene la condición física relacionada con la salud (10).

Diversos profesionales componen los equipos de salud. Entre ellos se encuentran los médicos, enfermeros, nutricionistas, kinesiólogos y profesores de educación física, entre otros. Por ejemplo, los médicos, son importantes en lo que respecta al asesoramiento sobre AF y, a su vez, una fuente de información muy respetada. Debería ser habitual la prescripción de AF por su parte. Esto no siempre 
sucede así pues existen barreras como la falta de formación e información en los estudios de grado, la escasa motivación y la poca realización de AF por ellos mismos. En Estados Unidos solo el 34 \% de los pacientes recibe información adecuada sobre AF (11). Cada profesional debería, cumplir su rol de prescripción de la AF y predicar con el ejemplo. Esto sería un importante aporte debido a que los profesionales de la salud son personas reconocidas, y sus consejos, en general, son tomados en cuenta. Resulta interesante, conocer la cantidad de AF y de tiempo sedentario realizada por quienes trabajan en ámbitos de salud. El objetivo de esta investigación fue cuantificar y calificar la actividad física y el tiempo sedentario realizada por quienes trabajan en ámbitos de salud, estudiando en ellos, además, marcadores metabólicos de riesgo como el colesterol, la glucemia en ayunas y el estado de nutrición.

\section{Método}

La investigación es de diseño epidemiológico, de tipo observacional, el manejo de datos es de corte transversal, siendo el nivel de análisis de datos de tipo analítico relacional. Se incluyeron en el trabajo 322 sujetos de ambos sexos, todos profesionales en el área de salud, que se desenvuelven en hospitales de la Ciudad Autónoma de Buenos Aires y el Gran Buenos Aires. El muestreo se realizó por conveniencia, y los encuestadores fueron profesionales médicos que recibieron entrenamiento sobre el uso del cuestionario. Se estudió el nivel de AF y la asociación entre el nivel de AF y su estado nutricional, el riesgo cardiovascular por colesterol total y el metabólico por glucemia plasmática.

Nivel de AF: el instrumento que se utilizó fue el Global Physical Activity Questionnaire - GPAQ (12) que indaga sobre las actividades realizadas en una semana habitual en los dominios trabajo, transporte y tiempo libre. Para categorizar los niveles de actividad física se tomó en cuenta el tiempo y la intensidad. En lo que respecta a intensidad se asignó a la actividad física de tipo moderado un valor de 4 METs (unidad de medida del índice metabólico) y al vigoroso de 8 METs; al transporte activo como caminar o andar en bicicleta se le clasificó como moderado (4 METs). Los valores de corte para AF vigorosa semanal fueron: 3 o más días de $\mathrm{AF} \geq 1500$ METs, o 7 de AF sumando $\geq 3000$ METs y para AF moderada semanal fueron: 60 minutos de AF vigorosa semanal o 150 minutos de actividad moderada o una combinación de 5 o más días alcanzando los 600 METs. El cuestionario permitió estudiar el tiempo sedentario, en minutos, y se utilizó el valor de 4 horas como punto de corte para considerarlo excesivo (13). La estatura fue medida con un estadiómetro deslizante de pared (Wiso ${ }^{\bullet}$, Brasil) con una precisión de $0.001 \mathrm{~m}$. El peso fue medido con una balanza de palanca (CAM modelo $\mathrm{P}-1001-\mathrm{P}$ ), con una precisión de $0.1 \mathrm{~kg}$. Se calculó el Índice de Masa Corporal (IMC), utilizando la fórmula peso (kg) / talla2 $\left(\mathrm{m}^{2}\right)$, y fue utilizado como una aproximación al estado nutricional. Se determinó colesterol total y glucemia en ayunas. Las variables demográficas estudiadas fueron Edad, Género y Profesión. Las categorías de Profesión fueron elegidas en razón que todos tienen contacto directo con los pacientes y podrían influir en sus decisiones de adoptar estilos de vida saludables. Se determinó colesterol total en $\mathrm{mg} / \mathrm{dl}$ : deseable (<200 mg/dl), intermedio alto (200 a $239 \mathrm{mg} / \mathrm{dl}$ ) y alto ( $\geq 240 \mathrm{mg} / \mathrm{dl}$ ) con los valores de cortes siguientes: Bajo $<200 \mathrm{mg} / \mathrm{dl}$ y Elevado $\geq 200 \mathrm{mg} / \mathrm{dl}$ (14). Se determinó la glucemia en ayunas en $\mathrm{mg} / \mathrm{dl}$ con los siguientes valores de corte: bajo (<70 mg/dl), normal (70 a 110mg/dl), elevado (11l a $126 \mathrm{mg} / \mathrm{dl}$ ) y muy elevado (>126mg/dl) (15).

Los datos fueron volcados en una planilla de Excel para Microsoft, presentando de este modo los datos de forma descriptiva. Para el estudio de normalidad de las variables se utilizó la prueba de Kolmogorov - Smirnov, determinándose que fue una muestra no normal. Se realizó un análisis de las variables en función de estudiar la AF con los marcadores para riesgo metabólico mediante el test de $\mathrm{Chi}^{2}$ de Pearson, nivel de significación de $p=0.05$, indagándose sobre el grado de asociación entre las variables dicotómicas mediante el coeficiente Phi o utilizando la prueba de V de Cramer para tablas mayores. Fue utilizada la prueba no paramétrica U de Mann-Whitney en el caso de variables cuantitativas e independientes. En cuanto a las consideraciones bioéticas, todos los sujetos fueron adultos y su participación fue voluntaria, utilizándose el consentimiento informado. Este estudio fue aprobado por el Comité Ético de Investigación de la Fundación Instituto Superior de Ciencias de la Salud, Código CISED 5/13.

\section{Resultados}

De los 322 sujetos estudiados, 221 fueron mujeres (68.6 \%) y 101 varones (31.4\%) entre los cuales se encontraron las siguientes profesiones: Médicos (41.3\%), Enfermeros/as (15.2\%), Nutricionistas (37.5\%) y Administrativos (6.2\%), todos pertenecientes al sistema de salud y en contacto con pacientes. La edad promedio de la muestra fue de $40.7 \pm 10.6$ años, con una edad mínima de 21 años y una edad máxima de 
72 años. De los 322 sujetos estudiados 196 (60.9\%) cumplen con los requisitos de AF suficiente, y 126 (39.1 \%) no lo hacen.

La Tabla $\mathrm{N}^{\circ} 1$ muestra que el $46.9 \%$ de los sujetos presenta un estado nutricional de sobrepeso u obesidad, que la glucemia era normal en la mayoría de los sujetos (93.5\%) y que el $68.9 \%$ de los sujetos presentan un colesterol óptimo.

Tabla 1. Factores de Riesgo Metabólico (n=322).

\begin{tabular}{|c|c|c|c|c|c|}
\hline \multicolumn{2}{|c|}{ Estado Nutricional } & \multicolumn{2}{|c|}{ Glucemia en ayunas (mg \%) } & \multicolumn{2}{|c|}{ Colesterol total (mg \%) } \\
\hline Categoría & Frecuencia (\%) & Categoría & Frecuencia (\%) & Categoría & Frecuencia (\%) \\
\hline Bajo peso & $7(2.2)$ & Bajo & $7(2.2)$ & Bajo & $222(68.9)$ \\
\hline Normopeso & $164(50.9)$ & Normal & $301(93.5)$ & Normal & $82(25.5)$ \\
\hline $\begin{array}{l}\text { Sobrepeso + } \\
\text { Obesidad }\end{array}$ & $151(46.9)$ & Elevado & $14(4.3)$ & Elevado & $18(5.6)$ \\
\hline
\end{tabular}

La Tabla $\mathrm{N}^{\circ} 2$ muestra que los sujetos con un nivel de AF suficiente poseen un alto porcentaje de niveles saludables de colesterol (73.5\%). Se estudió esta asociación mediante prueba de Chi-cuadrado de Pearson (valor de significación = 0.020), siendo débil la fuerza de la asociación (Phi = 0.086). Entre los niveles de actividad física y el estado nutricional se encontró una asociación estadísticamente significativa $(\mathrm{p}=0.041)$, luego se estudió la fuerza de la asociación mediante el estadístico Phi que la mostró débil (Phi = 0.094). Además, se estudió la relación entre los niveles de actividad física y la glucemia, que mostraron una relación no significativa con la prueba de $\mathrm{Chi}^{2}(p=0.358)$.

Tabla 2. Colesterol, Estado Nutricional y Actividad Física.

\begin{tabular}{|l|c|c|c|c|c|}
\hline & \multicolumn{2}{|c|}{ Colesterol } & \multicolumn{2}{c|}{ Estado nutricional } & \\
\hline $\begin{array}{c}\text { Actividad } \\
\text { física }\end{array}$ & $\begin{array}{c}\text { Normal } \\
\text { Frecuencia (\%) }\end{array}$ & $\begin{array}{c}\text { Elevado } \\
\text { Frecuencia (\%) }\end{array}$ & $\begin{array}{c}\text { Bajo o } \\
\text { Normopeso (\%) }\end{array}$ & $\begin{array}{c}\text { Sobrepeso u } \\
\text { obesidad (\%) }\end{array}$ & Total \\
\hline Insuficiente & $78(61.9)$ & $48(38.1)$ & $58(46)$ & $68(54)$ & 126 \\
\hline Suficiente & $144(73.5)$ & $52(26.5)$ & $113(57.7)$ & $83(42.3)$ & 196 \\
\hline Total & $222(68.9)$ & $100(31.1)$ & $171(53.1)$ & $151(46.9)$ & 322 \\
\hline
\end{tabular}

En la Tabla $\mathrm{N}^{\circ} 3$ se muestra la asociación entre los niveles de actividad física y la edad, encontrándose una asociación estadísticamente significativa $(p=0.004)$, la fuerza de la asociación se mostró débil (V de Cramer = 0.004).

Tabla 3. Edad (años) en relación con la Actividad Física.

\begin{tabular}{|l|c|c|c|c|c|c|c|}
\hline $\begin{array}{c}\text { Actividad } \\
\text { física }\end{array}$ & $<25$ & 25 a 34 & 35 a 44 & 45 a 54 & 55 a 64 & $>64$ & Total \\
\hline Insuficiente & $1(0.8 \%)$ & $28(22.2 \%)$ & $40(31,7 \%)$ & $33(26.2 \%)$ & $20(15.9 \%)$ & $4(3.2 \%)$ & $126(100 \%)$ \\
\hline Suficiente & $10(5.1 \%)$ & $63(32.1 \%)$ & $64(32.7 \%)$ & $46(23.5 \%)$ & $11(5.6 \%)$ & $2(1 \%)$ & $196(100 \%)$ \\
\hline Total & $11(3.4 \%)$ & $91(28.3 \%)$ & $104(32.3 \%)$ & $79(24.5 \%)$ & $31(9 \%)$ & $6(1.9 \%)$ & $322(100 \%)$ \\
\hline
\end{tabular}

En la Tabla $\mathrm{N}^{\circ} 4$ se estudia la asociación entre los niveles de actividad física y la profesión, encontrándose una asociación estadísticamente significativa ( $p=0.019)$, la fuerza de dicha asociación se mostró débil (V de Cramer $=0.019)$. Con respecto al tiempo sedentario se pudo constatar que el tiempo total promedio era de 347.83×195.26 minutos por día. En el ámbito laboral el tiempo dedicado 
a conducta sedentaria fue de $148.62 \pm 152.193$ minutos, en el transporte fue de $55.17 \pm 55.51$ minutos y en el tiempo libre el promedio fue de $144.04 \pm 97.66$ minutos. Por otro lado, el $67 \%$ de los sujetos reporto más de 4 horas de tiempo sedentario diario, con un máximo de 840 minutos. No resultaron significativas las relaciones entre el tiempo sedentario y los niveles de AF $(p=0.611)$, colesterol $(p=0.503)$, estado nutricional $(p=0.137)$ ni edad $(p=0.166)$ ni tiempo de actividad física moderada o vigorosa ( $U$ de Mann-Whitnney, $p=0.961)$.

Tabla 4. Profesión en relación con la Actividad Física.

\begin{tabular}{|l|c|c|c|c|c|}
\hline $\begin{array}{c}\text { Actividad } \\
\text { física }\end{array}$ & Médico & Enfermero & Nutricionista & Administrativo & Total \\
\hline Insuficiente & $61(48.4 \%)$ & $10(7.9 \%)$ & $48(38.1 \%)$ & $7(5.6 \%)$ & $126(100 \%)$ \\
\hline Suficiente & $72(36.7 \%)$ & $39(19.9 \%)$ & $72(36.7 \%)$ & $13(6.6 \%)$ & $196(100 \%)$ \\
\hline Total & $133(41.3 \%)$ & $49(15.2 \%)$ & $120(37.3 \%)$ & $20(6.2 \%)$ & $322(100 \%)$ \\
\hline
\end{tabular}

En la Tabla $\mathrm{N}^{\circ} 5$ se estudia la asociación entre el tiempo sedentario y la profesión, encontrándose una asociación estadísticamente significativa ( $p=0.009)$, aunque con una débil fuerza de asociación (V de Cramer = 0.009).

Tabla 5. Profesión en relación con el Tiempo Sedentario Diario.

\begin{tabular}{|l|c|c|c|c|c|}
\hline \multicolumn{1}{|c|}{ Tiempos Sed } & Médico & Enfermero & Nutricionista & Administrativo & Total \\
\hline$<4$ horas & $37(35.2 \%)$ & $10(9.5 \%)$ & $47(44.8 \%)$ & $11(10.5 \%)$ & $105(100 \%)$ \\
\hline$\geq 4$ horas & $96(44.2 \%)$ & $39(18 \%)$ & $73(33.6 \%)$ & $9(4.1 \%)$ & $217(100 \%)$ \\
\hline Total & $133(41.3 \%)$ & $49(15.2 \%)$ & $120(37.3 \%)$ & $20(6.2 \%)$ & $322(100 \%)$ \\
\hline
\end{tabular}

\section{Discusión}

En el presente estudio el $60 \%$ de los sujetos tiene un nivel de AF moderado o vigoroso suficiente, es decir, que cumplen con al menos 30 minutos por día y 5 días a la semana. El $40 \%$ no realiza la AF suficiente como para considerarse activos, encontrándose una disminución de la AF asociada a la edad. Este valor es ligeramente inferior al de la población general en Argentina, que es de 44.2\% de AF insuficiente según la $4^{\mathrm{a}}$ Encuesta Nacional de Factores de Riesgo (ENFR) (16). Que el personal de salud realice AF es importante, y en ese sentido deberían todos estar capacitados. Por ejemplo, la promoción de hábitos adecuados de AF durante la educación médica puede ser un paso importante para mejorar el asesoramiento preventivo de la AF que proporcionaran los futuros profesionales (17). Lo mismo sucede con los enfermeros o nutricionistas, o incluso con el personal administrativo que promociona los planes de salud médica. Con respecto a estudios sobre la actividad física de los profesionales de la salud, Marín Monroy y Aun Dau (18), interrogaron a 114 estudiantes de la Universidad Cooperativa de Colombia, sede Santa Marta (Población 383.991), mediante el cuestionario Healthy Doctor, con el cual se determinó la práctica de AF, reportando que el $96 \%$ de los estudiantes encuestados efectuaban algún tipo de AF diaria, el 51 \% realizaba una actividad vigorosa y el 43 \%, una actividad moderada. Además, el $65 \%$ opinaba que la facultad animaba a los estudiantes para que realizaran ejercicio y el 53 \% recibía información sobre AF. Entre los estudiantes, los niveles de AF son más altos en la Escuela de Medicina que en otros jóvenes estudiantes en la población general americana. Lobelo y colaboradores (19) realizaron una investigación abarcando 2316 estudiantes de medicina estadounidenses de 16 instituciones sobre sus niveles de AF mediante el cuestionario Godin. Se determinó que el 61 \% de ellos adhieren a las recomendaciones internacionales y el porcentaje de estudiantes que perciben que el asesoramiento en materia de AF y Salud sería altamente relevante para sus prácticas, disminuyó desde los primeros años de estudio al último del $69 \%$ a 53 \%. En Latinoamérica hay pocos estudios sobre la AF de los profesionales de la salud. En esa temática Giraldo, Echeverry, y Valencia (20) realizaron un trabajo sobre profesionales de la salud prestadores de servicios en el Sistema General de Seguridad Social en Salud de Colombia, utilizando el International Physical Activity Questionnaire o IPAQ. Este es un cuestionario que surgió como respuesta a la necesidad de crear un instrumento estandarizado 
para estudios poblacionales a nivel mundial a fines de los años 90. Por ese entonces, la aplicación de diferentes cuestionarios de evaluación de la AF dificultaba la comparación de resultados. Otro problema era la dificultad para valorar la AF en diferentes ámbitos. Existen varias versiones del instrumento de acuerdo al número de preguntas, el período y el método de aplicación (21). Fue el antecesor del GPAQ, el cuestionario utilizado en el presente estudio. Giraldo y colaboradores evaluaron un total de 57 profesionales de la Salud en Colombia, la mayoría de ellos de la ciudad de Pereira (población 358.681) y seleccionados de 10 diferentes EPS (Entidad Promotora de Salud). El $63 \%$ de los sujetos realizaba AF intensa o moderada, reconociendo los beneficios de la misma, pero solo el 37 \% la recomendaba en la consulta, siendo la falta de tiempo el mayor argumento para no prescribirla o recomendarla. El restante $43.9 \%$ no realizaba AF por falta de tiempo (20). Esto abre el interrogante sobre las dificultades para incorporar la prescripción de la AF en la consulta clínica diaria. Los niveles de actividad física del personal de salud han sido también estudiados en otras regiones del mundo, y por ejemplo en España Molina Aragonés y col. (22) han hecho notar que son sensiblemente distintos a los de la población general de referencia. En esta, el porcentaje de actividad física de intensidad leve y moderada es superior, siendo mediante la aplicación de la versión corta del Cuestionario internacional de actividad física y se estudiaron 544 sujetos. Un $26,5 \%$ de los individuos refirió un grado bajo de actividad física. El 31,5\% de los médicos manifestó un nivel de actividad bajo, 28.1 \% el personal auxiliar, enfermería el 24.7 \% y el personal de administración (19.0 \%).

En cuanto a los factores de riesgo metabólicos, el 50.9 \% del total de la muestra está en peso normal y $46.9 \%$ se encuentra con sobrepeso (37\%) u obesidad (9.9\%), valores por debajo de los registros de la $4^{\mathrm{a}}$ ENFR que informan un $66.1 \%$, según medidas objetivas (16). El estado nutricional y los niveles de AF muestran una asociación significativa, aunque leve, por lo que parecería que quienes realizan actividad física suficiente se encuentran con mejor peso que quienes no la realizan. Obviamente que es un factor coadyuvante, aunque no sea el determinante. Dado que el presente estudio no registra antecedentes en nuestro país, se consultaron fuentes extranjeras. Por ejemplo, Sanabria-Rojas y colaboradores (23) en su estudio sobre el "Nivel de AF en los trabajadores de una Dirección Regional de Salud de Lima, Perú" encontraron niveles de actividad menores a los recomendados. El estudio fue observacional y transversal, y abarcó a 172 trabajadores. Para el nivel de AF también se utilizó IPAQ. El estado nutricional fue evaluado a través del Índice de Masa Corporal, teniendo como resultado que el $88 \%$ de los sujetos tenían un nivel de AF bajo, valor muy diferente al presente estudio, y el exceso de peso era acusado por el 64.0 \%, cuando en Buenos Aires se encontró que así lo tenían el 46.9 \%. El autor sugiere como solución el establecimiento de un programa de prevención que incluya la AF vigorosa y una dieta saludable para disminuir la obesidad y el sobrepeso. Datos similares obtuvieron Cuti y colaboradores (24) sobre el estado nutricional y perfil lipídico en trabajadores administrativos del Hospital "Elezear Guzmán Barrón” de Trujillo (la ciudad más poblada del norte del Perú, con una población estimada de 799.550 habitantes), quien mediante un estudio correlacional transversal, evaluó a 64 trabajadores utilizando encuestas para datos personales y técnicas como antropometría y análisis de sangre. Encontró en su muestra un sobrepeso del 56.2 \% y una obesidad del 4.7 \% en los trabajadores de dicho hospital (60.9 \% con sobrepeso u obesidad, 14 puntos por sobre este estudio). Con respecto al colesterol, el $59.4 \%$ tenía un nivel de colesterol deseable, siendo en el presente estudio de $68.9 \%$, o sea casi un $10 \%$ mayor. En el presente estudio se encontró, además, una asociación entre los niveles de colesterol deseados y la AF, aunque con una débil fuerza de asociación. Se debe recordar que la AF regular de media o moderada intensidad favorece la movilización y uso de las reservas energéticas lipídicas produciéndose un aumento de las lipoproteínas de alta densidad (HDLcolesterol) y una disminución de las lipoproteínas de baja densidad (LDL_colesterol) (25).

Como ya fue mencionado, en el presente estudio los niveles de AF fueron efectivamente asociados a los niveles de colesterol, estado nutricional, pero también a la edad y al tipo de profesión, pero en todos los casos la asociación fue débil. Con respecto al estudio de la glucemia en ayunas, un $4.3 \%$ de sujetos reportó niveles elevados de glucemia, que se encuentra por debajo de la media reportada en la Encuesta Nacional de Factores de Riesgo para Enfermedades No Transmisibles (16). El tiempo sedentario informado fue elevado, con un promedio de casi seis horas, aunque hubo valores tan elevados como de 14 horas diarias. Se sabe que el tiempo sedentario representa un factor de riesgo independiente respecto de la insuficiente actividad física, y su impacto en salud puede ser estudiado a través de valoraciones objetivas como los marcadores cardio-metabólicos e inflamatorios como la glucemia, los triglicéridos, la proteína C-reactiva, la actividad de la lipoproteín lipasa, la insulina, la función de células Beta (HOMA—\%B) y la sensibilidad a la insulina (HOMA—\%S) (26). Se encontró que los médicos y nutricionistas fueron los más sedentarios. Es importante notar que el sujeto puede ser activo, cumplir con recomendaciones de actividad física, y también sedentario, si pasa varias 
horas de tiempo en conducta sedente. A su vez, se debe reconocer que las pausas activas podrían ser una herramienta útil para contrarrestar este fenómeno, y que su implementación en ámbitos de la salud debiera ser normativa. El propio gobierno argentino así lo entiende y dispone de herramientas digitales para la promoción de esta actividad (27), propuesta a la que se han hecho eco las universidades implementando este tipo de programas entre estudiantes y docentes (28).

Las limitaciones que se encontraron en este estudio fueron las propias de trabajar con una encuesta, las cuales poseen la subjetividad de los encuestados respecto a las respuestas dadas en cuanto al tiempo sedentario o el tiempo que pasan realizando AF y la intensidad con la que la realizan. Pero son económicas, y aun aceptando sus limitaciones, todavía resultan costo-eficaces para estudios poblacionales.

\section{Conclusión}

Aproximadamente el $60 \%$ del grupo de profesionales de la salud de Buenos Aires estudiado realiza AF moderada y vigorosa como recomienda la OMS para la prevención de las enfermedades no transmisibles. Y quienes realizan AF suficiente parecen beneficiarse con estados nutricionales y metabólicos adecuados. En definitiva, la AF en este grupo de profesionales es mayor que en la población general, y quienes la realizan gozan de múltiples beneficios. Pero un $40 \%$ de los sujetos estudiados no alcanza los niveles de suficiente AF, en detrimento de su propia salud. Además, la conducta sedentaria es elevada y reportada en todos los dominios. Con el agravante de que representa un factor de riesgo independiente de si se es activo o poco activo. Se podría recomendar implementar políticas que contribuyan a mejorar los niveles de AF, disminuyendo el tiempo sedentario (en lo laboral con pausas activas, por ejemplo) entendiendo que son ellos los ejemplos en la promoción de hábitos para mejorar la calidad de vida.

Fuente de financiamiento: autogestión.

\section{Referencias bibliográficas}

Piercy KL, Troiano RP, Ballard RM, et al. The Physical Activity Guidelines for Americans. JAMA. 2018; 320(19): 2020-8. Márquez RS, Rodríguez OJ, Olea S. Sedentarismo y salud: Los efectos beneficiosos de la actividad física. Apunts. Educación física y deportes. 2006;83:12-24.

American College of Sports Medicine. ACSM's Guidelines for Exercise Testing and Prescription. 10a. ed. Philadelphia, Pennsylvania: Lippincott Williams \& Wilkins, 2019.

Organización Mundial de la Salud. Actividad física (sitio web). 2020. Recuperado de: http://www.who.int/mediacentre/factsheets/fs385/es/ Colberg SR, Sigal RJ, Yardley JE, et al. Physical Activity/Exercise and Diabetes: A Position Statement of the American Diabetes Association. Diabetes Care. 2016; 39(11): 2065-79.

American Diabetes Association. Facilitating Behavior Change and Well—being to Improve Health Outcomes: Standards of Medical Care in Diabetes-2020. Diabetes Care. 2020; 43(Supplement1): S48-S65

Organización Mundial de la Salud. Inactividad física: un problema de salud pública mundial. Estrategia mundial sobre régimen alimentario, actividad física y salud. (sitio web). 2019. Recuperado de: https://www.who.int/dietphysicalactivity/factsheet_inactivity/es/ Leiva AM, Martínez MA, Cristi-Montero C, et al. El sedentarismo se asocia a un incremento de factores de riesgo cardiovascular y metabólicos independiente de los niveles de actividad física. Revista médica de Chile. 2017; 145(4): 458—67.

Mojáiber RB. El Sedentarismo, factor de riesgo contrario a la esencia humana. Revista Cubana de Medicina General Integral. 1998; 14(3): $211-2$.

Samaniego VP, Devis JD. La promoción de la actividad física relacionada con la salud. La perspectiva de proceso y de resultado. Revista Internacional de Medicina y Ciencias de la Actividad Física y el Deporte. 2003; (3):69-74.

Lobelo F, Duperly J, Frank E. Physical activity habits of doctors and medical students influence their counselling practices. British Journal of Sports Medicine. 2009; 43(2):, 89-92.

World Health Organization. Global Physical Activity Surveillance. Chronic diseases and health promotion. (sitio web). 2016. Recuperado de: http://www.who.int/chp/steps/GPAQ/en/

Ekelund U, Steene-Johannessen J, Brown WJ, et al. Does physical activity attenuate, or even eliminate, the detrimental association of sitting time with mortality? A harmonised meta—analysis of data from more than 1 million men and women. Lancet. 2016; 388(10051): 1302-10.

American Heart Association. Qué significan mis niveles de colesterol? Respuestas del corazón (sitio web). 2012. Recuperado de: https:// www.heart.org/idc/groups/heart_public/@wcm/@hcm/documents/downloadable/ucm_316249.pdf Benzadón M, Forti L, Sinay I. Actualización en el diagnóstico de la diabetes. Medicina. Buenos Aires. 2014; 74(1):64—8. 


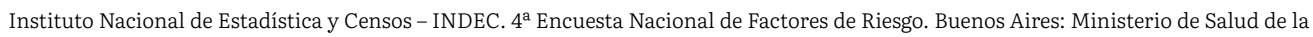
Nación; 2019. ISBN 978-950-896-554-7

Gates AB. Training tomorrow's doctors, in exercise medicine, for tomorrow's patients. Br J Sports Med. 2015; 49: $207-8$.

Marín Monroy C, Aun Dau F. Actividad física y estudiantes de medicina en Santa Marta. Revista Memorias. 2010; 8(14): 24-30.

Lobelo F, Frank E, Tong E, Carrera J, Duperly J. Physical activity levels and counseling practices of U.S. medical students. Medicine and Science in Sports and Exercise. 2008; 40(3): 413-21.

Giraldo JC, Echeverry PG, Valencia CDZ. Caracterización de una muestra de profesionales de la salud prestadores de servicios de salud en el SGSS, con relación a la práctica de la actividad física. Revista Médica de Risaralda. 2005; 11(2):1-17.

Mantilla Toloza SC, Gómez-Conesa A. El Cuestionario Internacional de actividad física. Un instrumento adecuado en el seguimiento de la AF poblacional. Revista Iberoamericana de Fisioterapia y Kinesiología. 2007; 10(1): 48-52.

Molina Aragonés JM, Sánchez San Cirilo S, Herreros López M, Vizcarro Sanagustín D, López Pérez C. Medicina de Familia. SEMERGEN. Prevalencia de actividad física en profesionales de atención primaria de Cataluña. 2017 Julio — Agosto;Vol. 43(5):352-7. DOI: 10.1016/j. semerg.2016.04.026

Sanabria-Rojas H, Tarqui-Mamani C, Benavides WP, Zaldívar HP, Castillo LM. Nivel de actividad física en los trabajadores de la Dirección Regional de Salud de Lima, Perú. Revista de Salud Pública. 2014, 16(1): 53—62.

Cuti G, Leny B, Velezmoro H, Sintia P. Estado nutricional y perfil lipídico, en trabajadores administrativos del hospital Elezear Guzmán Barrón, Chimbote. (Tesis de Licenciatura) Trujillo, Perú: Universidad privada Cesar Vallejo, Facultad de Ciencias Médicas. Escuela Académico Profesional de Nutrición; 2010.

Rojo—González JJ. Un análisis de la actividad física para la salud. Revista Encuentros Multidisciplinares. 2003 mayo—agosto; 14: 1—9.

Healy GH, Matthews CE, Dunstan DW, Winkler EAH, Owen N. Sedentary time and cardio-metabolic biomarkers in US adults: NHANES 2003-06. European Heart Journal. 2011; 32(5): 590-7.

Universidad Nacional de Córdoba. Pausa activa. (sitio en la web). Accesible en: https://www.unc.edu.ar/vida—estudiantil/pausa—activa 